\title{
Identificando a Paralisia do Sexto Nervo Ótico em Vídeos Através de Redes Neurais Convolucionais e Curvatura Isophotes
}

\author{
Polyana B. Costa ${ }^{1}$, João Dallyson S. Almeida ${ }^{1}$, Jorge Antônio Meireles Teixeira ${ }^{2}$, \\ Geraldo Braz Júnior ${ }^{1}$, Anselmo Cardoso de Paiva ${ }^{1}$ \\ ${ }^{1}$ Núcleo de Computação Aplicada - Universidade Federal do Maranhão (UFMA) \\ São Luís - MA - Brasil \\ ${ }^{2}$ Departamento de Medicina I. - Universidade Federal do Maranhão (UFMA) \\ São Luís - MA - Brasil \\ \{polyanabcosta, jdallyson, jorgemeirelesl,ge.braz, anselmo.c.paiva\}@gmail.com
}

\begin{abstract}
This article describes a methodology to aid in the identification of the sixth nerve palsy using videos. Since the usual techniques for diagnosing this paralysis are invasive or expensive, a non-invasive and affordable method could be helpful, supporting the ophthalmologist diagnosis. The proposed method uses the convolutional neural network YOLO to detect the eyes in a face and the Isophotes Curvature to find the center of the eye. Both techniques are applied in each frame of a video in which the patient moves its eyes, keeping track of the eye movement in order to calculate the average speed in which each eye moves. The obtained results showed that the eyes that have the paralysis move $42 \%$ slower than a healthy eye.
\end{abstract}

Resumo. Este artigo descreve uma metodologia para auxiliar na identificação da paralisia do sexto nervo utilizando vídeos. Uma vez que os métodos usuais para diagnóstico dessa paralisia são invasivos ou caros, um método não invasivo e acessível pode ser útil para apoiar ou guiar o diagnóstico do oftalmologista. O método proposto neste trabalho usa a rede neural convolucional YOLO para detectar a região dos olhos em faces e a curvatura de Isophotes para encontrar o centro dos olhos em cada quadro do vídeo, mantendo assim um registro do movimento dos olhos durante um período de tempo, a fim de calcular a velocidade média com que cada olho se move. Os resultados obtidos mostraram que um olho que tem paralisia se move em média de forma $42 \%$ mais lenta que um olho saudável.

\section{Introdução}

O sexto nervo craniano, também conhecido como nervo abducente, é um nervo muito importante que está diretamente ligado à contração do músculo reto lateral [Ehrenhau 2018]. Este músculo permite que o olho vire para o seu canto externo, possibilitando que a pessoa olhe para a esquerda ou direita. A paralisia deste nervo impede que alguns dos músculos que controlam o movimento dos olhos funcionem adequadamente [GARD 2015]. Além disso, o mau funcionamento do nervo pode causar dor de cabeça, enxaqueca, visão turva, vertigem e visão dupla (diplopia) quando a pessoa tenta movimentar o olho em direção ao canto externo [Rubin and Cornell 2018] [Curi et al. 2013]. 
A paralisia do sexto nervo é considerada uma disfunção rara; estudos feitos em crianças norte-americanas mostraram que ocorrem 2.5 casos a cada 100 mil habitantes [Ehrenhau 2018]. O diagnóstico deve ser feito cedo, pois quando a causa é tratada, a paralisia geralmente desaparece. A figura 1 mostra o sexto nervo e o músculo reto lateral.

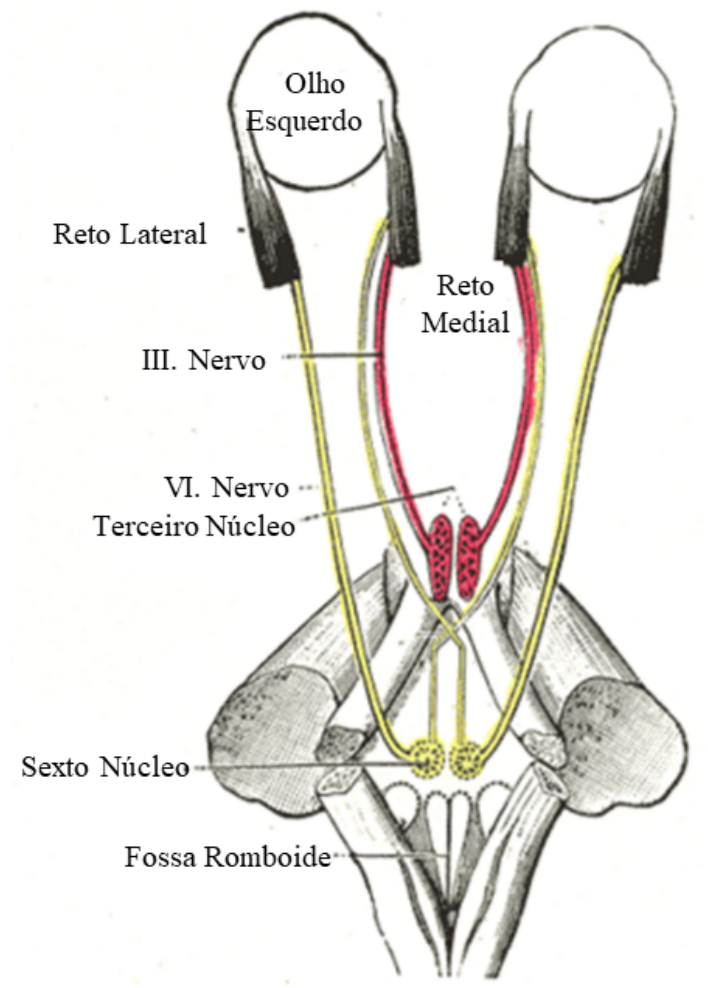

Figura 1. Sexto nervo e reto lateral.

Adaptado de [Gray and Goss 1974].

A avaliação clínica e neurológica é essencial no diagnóstico etiológico e tratamento adequado. A avaliação oftalmológica visa observar alterações motoras, suas consequências no sistema oculomotor, sua evolução, a fim de minimizar os sintomas durante a evolução da doença e evitar sequelas [Curi et al. 2013]. Existem duas maneiras principais de detectar a paralisia do sexto nervo, que são o Teste de Geração de Força e a Eletro-oculografia.

De acordo com Curi et al. (2013), o Teste de Geração de Força (FGT) explora o potencial da força muscular e pode diferenciar entre paralisia parcial ou total. É um teste simples e confiável, mas o resultado depende da experiência do examinador. Após a anestesia tópica, um fórceps é anexado ao membro temporal do olho lesionado, o olho é levado para a adução e o paciente é solicitado a mover rapidamente o olho em direção ao músculo reto lateral examinado, fazendo com que o examinador sinta a força desse músculo no fórceps. A quantidade de força sentida é então analisada. Muitas vezes a força é tão forte que causa uma ruptura da conjuntiva.

A eletro-oculografia (EOG) é um exame que registra os movimentos oculares durante os testes eletronistagmográficos. É baseado no potencial corneorretiniano. Para me- 
dir e acompanhar os movimentos dos olhos, eletrodos são colocados ao redor dos olhos; quando os movimentos se aproximam dos eletrodos, uma diferença de potencial é registrada para ser usada como uma medida para a posição do olho [Martinsen and Grimnes 2011].

Ambas as técnicas são eficazes na detecção da paralisia do sexto nervo, mas o FGT é um procedimento invasivo e dependente da experiência do oftalmologista, e o EOG é um exame dispendioso. A fim de apoiar ou orientar o diagnóstico do médico com um método não invasivo e acessível, este trabalho visa propor um método para a detecção automática da paralisia do sexto nervo usando vídeos, obtendo a velocidade com que cada olho se move durante um período de tempo. Os passos iniciais da metodologia e os resultados preliminares serão apresentados, a fim de determinar se a metodologia proposta é promissora. As técnicas aplicadas serão explicadas nas seções seguintes.

\section{Fundamentação Teórica}

Nesta seção, serão apresentadas todas as técnicas utilizadas na metodologia deste trabalho.

\subsection{Curvatura de Isophotes}

Tendo em vista que os olhos são caracterizados por padrões de brilho simétricos radialmente, Valenti e Gevers (2008) propuseram uma nova abordagem para localização do centro ocular baseada na observação desses padrões. O método proposto pelos autores utiliza Isophotes para inferir o centro de padrões semi-circulares de imagens de baixa resolução, atingindo uma acurácia de aproximadamente $98 \%$ na localização do centro do olho. De forma resumida, a curvatura de um Isophotes pode ser calculada como mostra a equação 1 .

$$
k=-\frac{L_{v v}}{L_{w}}
$$

Onde, $L_{v v}$ é descrita na equação 2:

$$
L_{v v}=L_{y}^{2} L_{x x}-2 L_{x} L_{x y} L_{y}+L_{x}^{2} L_{y y}
$$

E $L_{w}$ na equação 3:

$$
\left(L_{x}^{2}+L_{y}^{2}\right)^{\frac{3}{2}}
$$

$L(x, y)$ é a função de luminância; $L_{x}$ e $L_{y}$ são as derivadas de primeira ordem da imagem em $x$ e em $y$ e $L_{x x}, L_{x y}$ e $L_{y y}$ são as derivadas de segunda ordem da imagem [Gonzalez and Woods 2007], o $k$ resultante é um mapa de curvatura. A fim de encontrar os centro do círculos presentes no mapa de curvatura, é preciso calcular os vetores de deslocamento que representam a posição estimada dos centros, que podem ser mapeadas em um acumulador. Os vetores de deslocamento são definidos na equação 4:

$$
D(x, y)=\frac{\left\{L_{x}, L_{y}\right\}\left(L_{x}^{2}+L_{y}^{2}\right)}{L_{y}^{2} L_{x x}-2 L_{x} L_{x y} L_{y}+L_{x}^{2} L_{y y}}
$$

Em seguida, é necessário calcular a curvatura da forma, que não deve ser confundida com $k$, que é o mapa de curvaturas Isophotes. A curvatura da forma é apresentada na equação 5:

$$
\text { Curvatura }=\sqrt{L_{x x}^{2}+2 L_{x y}^{2}+L_{y y}^{2}}
$$


Após obter os vetores de deslocamento e a curvatura, é preciso limitar as curvaturas que se deseja, definindo um raio mínimo e um raio máximo, a fim de descartar pixels que possuem curvatura muito baixa (pixels pertencentes a linhas) ou curvatura muito alta (pi$x e l s$ pertencentes a circulos muito pequenos). Após isso, constroi-se um mapa de centros, onde verifica-se se para cada pixel, o vetor de deslocamento correspondente possui valor entre os limites de raio mínimo e máximo definido, caso possua, o pixel naquela posição receberá o valor da curvatura na respectiva posição, formando um acumulador de valores. Em seguida, a fim de saber o centro com o maior acumulador, procura-se a posição do mapa de centros com o maior valor, esse será o centro do círculo.

\subsection{YOLO}

YOLO, sigla para You Only Look Once (Você só olha uma vez) é uma rede neural convolucional para detecção de objetos em tempo real, com uma nova abordagem para detecção proposta por [Redmon et al. 2016]. Em vez de tratar a detecção de objetos como um problema de classificação, YOLO a vê como um problema de regressão. YOLO consiste em um modelo unificado em que uma única rede prevê a região em que um objeto está enquanto informa a qual classe o objeto detectado pertence. Isso faz com que YOLO seja extremamente rápida, sendo executada em até 45 quadros por segundo (FPS) em sua versão convencional e até 155 (FPS) em sua versão reduzida.

A rede oferece 3 dados como saída: a primeira contém as coordenadas da bounding box encontrada, a sua confiança e a classe do objeto encontrada dentro desta região. A confiança da bounding box encontrada é calculada com base no IOU bounding box com a bounding box correta (ground truth) [Rosebrock 2016].

\subsubsection{Arquitetura da Rede}

A arquitetura usada no desenvolvimento deste trabalho é a YOLOv3, a terceira versão da YOLO proposta em [Redmon and Farhadi 2018] com implementação no Keras por [Trade 2018]. Nessa versão, também conhecido como DarkNet 53, a rede possui blocos de convolução e blocos residuais semelhantes aos da [Szegedy et al. 2017]. O primeiro bloco de convolução utilizado, aqui chamado de DarkConv, faz uma convolução em 2D com número $\mathrm{N}$ de filtros e kernel de tamanho k, seguido por uma camada de Batch Normalization, seguida pela função de ativação LeakyReLu (Leaky Rectified Linear Activation) [Redmon et al. 2016], calculada como mostrado na equação 6.

$$
\phi=\left\{\begin{array}{cc}
\mathrm{x}, & \text { se } \mathrm{x}>0 \\
0.1 \mathrm{x}, & \text { caso contrário }
\end{array}\right\}
$$

O bloco residual utilizado nessa rede é similar ao proposto em [Szegedy et al. 2017], aqui chamado de DarkBlock. O bloco recebe como entrada um bloco do tipo DarkConv de kernel $3 x 3$, N filtros e stride 2, que é somado o seu resultado após passar por dois outros blocos DarkConv, o primeiro com kernel $1 x 1, \frac{N}{2}$ filtros e o segundo com kernel $3 x 3$, e $N$ filtros. O terceiro bloco, chamado aqui de LastLayers, consiste em 5 
blocos do tipo DarkConv, alternando em blocos de kernel $1 x 1$ e $N$ filtros, com blocos de kernel $3 x 3$ e $N$ x 2 filtros. O bloco final da rede, chamado de YOLO Output é responsável pela saída da rede, e consiste em um bloco do tipo DarkConv de kernel $3 x 3$ e $N$ x 2 filtros, seguido de uma camada de convolução 2D sem função de ativação definida, funcionando como uma ativação linear. Esta última convolução é responsável pela parte de Regressão da rede, possuindo kernel $1 x 1$, e um número $S$ de filtros, que é a multiplicação do número de âncoras pela soma do número de classes mais 5, ou seja, $S=9 \times(1+5)$.

A Figura 2 mostra a arquitetura completa da rede. O corpo da rede possui 24 blocos de convolução, resultando em 47 camadas de convolução, e com a primeira saída da rede têm-se 53 camadas; (por isso o nome DarkNet 53 [Redmon and Farhadi 2018]. São fornecidas 3 saídas porque o resultado da rede é predito em 3 escalas diferentes.

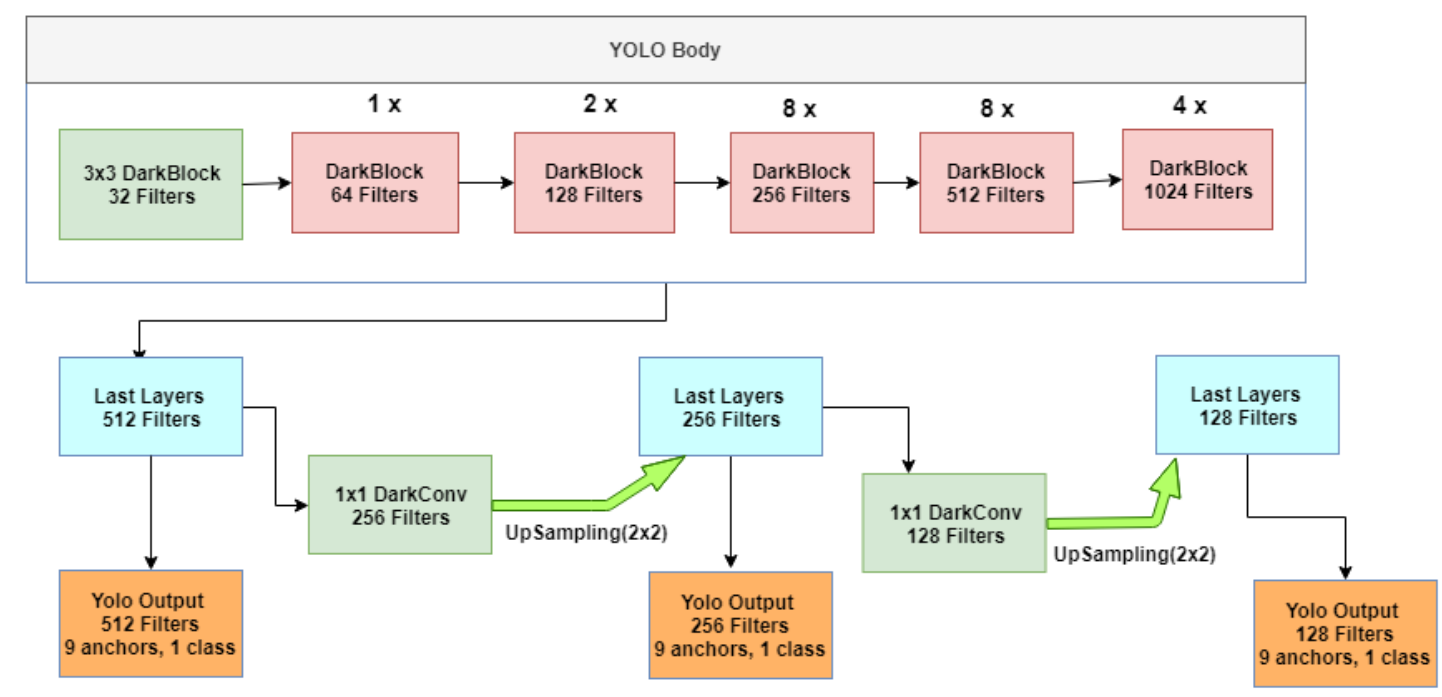

Figura 2. Arquitetura da rede utilizada.

\section{Metodologia}

A metodologia proposta neste trabalho utiliza a rede neural convolucional YOLO para identificar a região dos olhos em uma face e em seguida faz o cálculo da curvatura Isophotes sob cada imagem de olho retornado pela YOLO, obtendo-se o centro do olho. Ambas as técnicas são aplicadas em cada frame de um vídeo, a fim de manter um registro da movimentação de cada olho em um intervalo de tempo, para posteriormente calcular a velocidade média com que cada olho se moveu durante o vídeo. A rede YOLO foi escolhida por sua rapidez em execução, e a técnica de Isophotes foi escolhida por sua eficiência na detecção de centro de círculos em imagens de baixa resolução, e por não ser uma técnica densamente parametrizada. A Figura 3 ilustra cada etapa da metodologia, que serão explicadas nas subseções a seguir.

\subsection{Aquisição dos Vídeos e Pré-Processamento}

A metodologia desenvolvida neste trabalho foi aplicada em vídeos de pacientes que possuem a paralisia do sexto nervo, bem como em alguns pacientes saudáveis. Os vídeos foram fornecidos por um oftalmologista que colaborou com este trabalho, que inclusive 


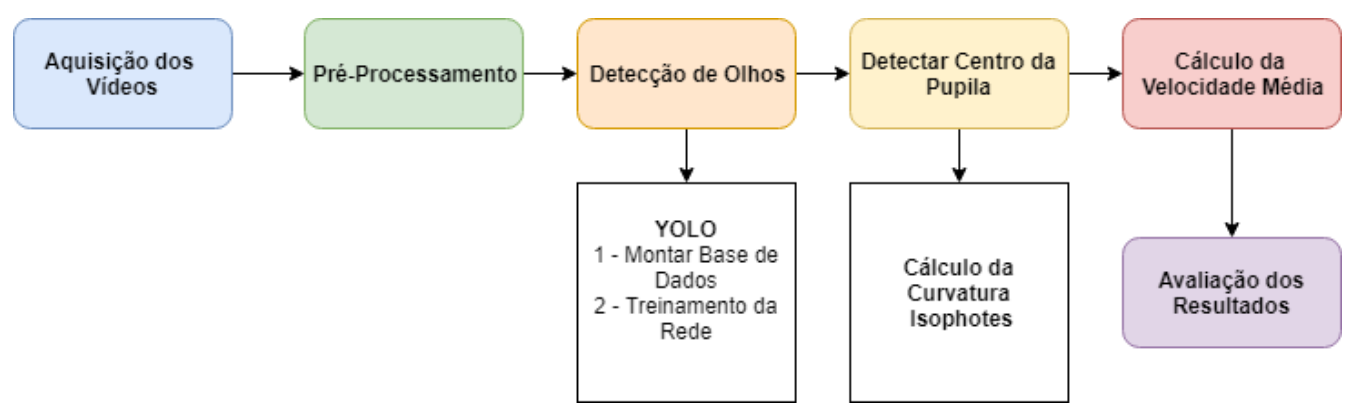

Figura 3. Etapas do método proposto.

sugeriu o desenvolvimento do mesmo; o próprio especialista gravou seus pacientes movimentando ambos os olhos para a esquerda, direita, para cima e para baixo. Ao todo, foram usados 8 vídeos de 7 pacientes diferentes, sendo que deste número, 6 pacientes possuem a paralisia e 2 são saudáveis. Para um dos pacientes gravou-se o vídeo antes e depois da cirurgia para correção da paralisia do sexto nervo. Não foi definido protocolo para aquisição dos vídeos, visto que o oftalmologista os gravou sem maiores pretensões, portanto em alguns deles foi necessário fazer um pré-processamento. Entretanto, em todos os vídeos, os rostos dos pacientes estão centralizados, mas como não foi usado tripé, em alguns frames a imagem foi desestabilizada.

Já que método de Isophotes funciona melhor em imagens degradadas ou de baixa resolução, alguns vídeos foram redimensionados, e outros tiveram algumas partes cortadas, como partes em que o paciente não aparecia. O baixo número de vídeos é devido a raridade da patologia entre os pacientes atendidos pelo oftalmologista em questão. Cada frame ou quadro de cada um dos vídeos obtidos foi utilizado na fase seguinte da metodologia, a detecção de olhos.

\subsection{Detecção de Olhos}

A detecção de olhos foi feita através da rede YOLO. Essa rede foi escolhida por sua rapidez e eficiência na detecção de objetos. Uma base de dados privada com 243 imagens de rosto inteiro, totalizando 486 olhos, foi utilizada para treinamento e validação da rede YOLO. A base foi fornecida pelo mesmo oftalmologista local que forneceu os vídeos mencionados na seção anterior, contendo imagens de 45 pacientes diferentes, com 5 ou 6 imagens de cada paciente olhando para diferentes direções, como para a frente, direita, esquerda etc. A base é composta por imagens de alta resolução, com 1572x2048 pixels.

Para treinamento da CNN utilizada, fez-se aumento dos dados da base. Cada imagem foi espelhada, rotacionada em 90, 180 e 270 graus, gerando 4 novas imagens. Depois do aumento dos dados, a base passou a ter 1215 imagens. Dessas imagens, 1095 foram usadas para treino, e 120 para validação. As imagens da validação foram separadas por paciente, para que não houvesse imagens do mesmo paciente durante treino e validação. As bounding boxes correspondentes à posição de cada olho foram definidas com base na marcação (Ground Truth) da íris de cada olho. Em seguida, um arquivo de texto contendo o caminho para cada imagem, as bounding boxes encontradas na referida imagem, bem como a classe de cada uma delas foi gerado e usado no treinamento da rede, e um arquivo similar foi usado na validação. Usou-se somente esta base porque as imagens apresentam o rosto inteiro do paciente e possui-se a marcação da posição dos olhos, futuramente 
usada para avaliação do método de Isophotes.

A rede utilizou pesos pré-treinados da ImageNet [Deng et al. 2009], e foi treinada em 100 épocas de duas maneiras diferentes. Na primeira abordagem, todas as camadas entre o primeiro bloco DarkBlock e o bloco YOLO Output foram congeladas nas primeiras 50 épocas, sendo descongeladas da época 50 a 100 . O que faz com que os pesos dessas camadas não sejam atualizados, o que acelera o treinamento. Na segunda abordagem não houve congelamento de nenhuma camada em nenhuma das épocas. A abordagem com congelamento obteve os melhores resultados, que serão apresentados na seção 4. Após o treinamento, o modelo obtido foi usado para a detecção de olhos em cada frame dos vídeos utilizados, identificando bounding boxes referentes às regiões que contém olhos. Cada região obtida foi analisada para determinar se pertencia ao olho esquerdo ou olho direito; os vídeos possuem imagens centralizadas da face do paciente, portanto, considerou-se olho esquerdo aquele que estivesse até a metade da imagem, e olho direito seria aquele que estivesse a partir da metade. Somente a região que contém cada olho foi passada individualmente para a próxima fase.

\subsubsection{Detecção do Centro da Pupila}

A detecção do centro da pupila foi feita através do cálculo da curvatura de Isophotes. Esta técnica foi escolhida por não ser densamente parametrizada, e por apresentar alta acurácia da detecção de centro de círculos. A imagem obtida na etapa anterior foi usada em níveis de cinza, e como pré-processamento, efetuou-se uma suavização da imagem através da Gaussiana; verificou-se que ao utilizar um kernel 3x3, a estimação do centro não retornou resultados satisfatórios, já que muitos pontos de brilho ainda permaneceram na imagem. Portanto, usou-se uma Gaussiana com kernel 9x9. Após esse pósprocessamento, calculou-se o mapa de de curvaturas $k$ mostrado na Equação 1, os vetores de deslocamento mostrado na Equação 4, e a magnitude dos vetores de deslocamento, a fim de usar esses três critérios para montar a matriz de votação, que utiliza como peso do voto a curvatura do pixel apresentada na Equação 5. Os vetores de deslocamento servem para indicar o centro da forma a que determinado pixel pertence. Portanto, o centro de um círculo vai aparecer como centro de todos os pixels que constituem a circunferência do círculo, tendo uma grande quantidade de votos. Essa matriz de votos forma o mapa de centros, o pixel de maior valor nesse mapa deverá ser o centro do círculo. Os únicos parâmetros a serem definidos são os raios mínimo e máximo, que são usados para rejeitar círculos muito grande ou pequenos. Após alguns testes, os valores utilizados foram para raio mínimo e máximo ficaram entre 2 e 20 , respectivamente. A figura 4 mostra cada etapa do método de Isophotes.

O centro do círculo referente à pupila é calculado em cada frame, a fim de se manter um registro da trajetória da movimentação de cada olho durante o vídeo. Esse registro é usado na etapa a seguir.

\subsection{Cálculo da Velocidade Média}

De acordo com [Resnick et al. 2001], uma das formas mais comuns de descrever o movimento de um objeto é através da sua posição em função do tempo, para isso, pode-se calcular a velocidade escalar média. Ela é a razão entre o deslocamento $(\Delta S)$ e o tempo de 
(a)

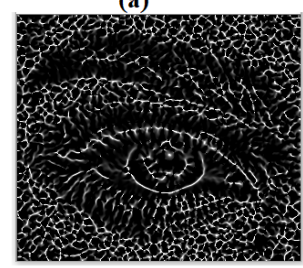

(b)

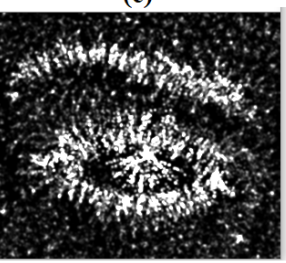

(d)

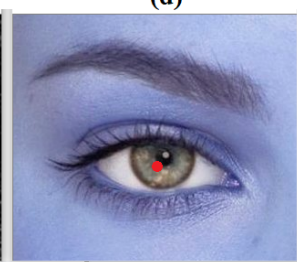

Figura 4. Etapas do Isophotes. (a) Mapa de Curvaturas Isophotes. (b) Vetores de deslocamento. (c) Mapa de centros. (d) Centro de maior valor (ponto vermelho).

duração do deslocamento $(\Delta T)$, e pode ser descrita através da Equação 7; para o cálculo de $(\Delta S)$ deve-se considerar as posições inicial e final, e para o cálculo de $(\Delta T)$ deve-se levar em conta o tempo inicial e final. No método proposto, calculou-se a velocidade apenas em relação ao eixo X. Neste caso, a posição inicial é a menor posição em X (canto interno do olho) durante um intervalo de tempo $\delta T$, e a posição final é a maior posição em X (canto externo). Os conceitos de tempo inicial e final foram dados em função da quantidade de frames do vídeo utilizado.

$$
\text { Velocidade }_{\text {media }}=\frac{\Delta S}{\Delta T}=\frac{\text { Pos }_{\text {Final }}-\text { Pos }_{\text {Inicial }}}{\text { Tempo }_{\text {Final }}-\text { Tempo }_{\text {Inicial }}}
$$

\section{Resultados}

Cada técnica aplicada neste trabalho foi avaliada de forma individual. Para analisar o desempenho geral da rede, usou-se a precisão. De acordo com [Powers 2011], a precisão apresenta uma razão entre os objetos que foram detectados corretamente (VP - Verdadeiros Positivo), pela soma dos VP, com os objetos que foram detectados incorretamente, FP - Falsos Positivo. A rede foi testada numa base de 60 imagens que contém o rosto inteiro, obtidas durante o exame de análise do reflexo vermelho, também fornecidas por um oftalmologista local. A Tabela 1 mostra os resultados da precisão.

Tabela 1. Resultado da Precisão da detecção em ambas as abordagens.

\begin{tabular}{|c|c|c|c|c|}
\hline Abordagem & Olhos Existentes & VP & FP & Precisão \\
\hline YOLOV3 & 120 & 120 & 5 & $96 \%$ \\
\hline
\end{tabular}

Para avaliar o resultado do método da curvatura de Isophotes, utilizou-se $20 \%$ das imagens da base mencionada em 3.2, totalizando 48 imagens e 96 olhos. A métrica utilizada foi a porcentagem do erro, proposta em Ouabida et al. 2017. O autor divide o valor obtido pelo método deles pelo valor correto da posição do centro dos olhos, a fim de saber a porcentagem do erro entre esses dois valores. A mesma operação foi feita para cada imagem, em relação aos valores de centro em $x$ e em $y$. No fim, foi obtida a média das porcentagens para cada critério a ser analisado; o resultado é apresentado na Tabela 2.

Para analisar o resultado do método como um todo, calculou-se a velocidade média do deslocamento de cada olho em cada vídeo da base utilizada. A tabela 3 apre- 
Tabela 2. Porcentagem do erro da posição do centro do olho.

\begin{tabular}{|c|c|c|}
\cline { 2 - 3 } \multicolumn{1}{c|}{} & Erro em X & Erro em Y \\
\hline Isophotes & 0,084 & 0,052 \\
\hline
\end{tabular}

senta os resultados para cada olho. Os olhos marcados como doente são os que possuem a paralisia.

Tabela 3. Velocidade média com que cada olho se move. (A velocidade é dada pela razão entre a distância percorrida em pixels pela quantidade de quadros)

\begin{tabular}{|c|c|c|}
\hline Video & Velc. Olho Direito & Velc. Olho Esquerdo \\
\hline 1 & 0,103 (doente) & 0,342 \\
\hline 2 & 0,322 & 0,404 \\
\hline 3 & 0,301 (doente) & 0,573 \\
\hline 4 & 0,156 & 0,185 \\
\hline 5 & 0,617 & 0,364 (doente) \\
\hline 6 & 0,201 & 0,128 (doente) \\
\hline 7 & 0,277 & 0,189 (doente) \\
\hline 8 & 0,092 & 0,028 (doente) \\
\hline
\end{tabular}

Ao observar a velocidade média entre cada olho, percebe-se que o olho doente se move de forma mais lenta que o olho saudável, apresentando quase a metade da velocidade do olho que não possui paralisia. Em média, a velocidade média do olho doente é $42 \%$ menor que a do olho saudável. Nas linhas 2 e 4 da tabela, referentes à pacientes que possuem os dois olhos saudáveis, verifica-se que a diferença entre a velocidade dos dois olhos é bem menor, o que corrobora a conclusão de que o olho que possui paralisia se move mais devagar, além de apresentar um deslocamento bem menor em relação ao eixo $\mathrm{X}$, conforme ilustra a Figura 5. Percebe-se que o deslocamento em relação ao eixo X do olho esquerdo (olho doente) é uma pouco maior que 20 pixels, enquanto que o olho direito (saudável) chega a 80 pixels. Ao analisar a velocidade e pixels por frame do mesmo vídeo, como mostra a Figura 6, percebe-se que enquanto o olho saudável (direito) se move cerca de 3 pixels a cada frame, o olho doente se move menos que a metade disso, chegando a 2 pixel por frame no intervalo mostrado na figura.

\section{Conclusão}

Este trabalho apresentou uma metodologia para auxílio na identificação da paralisia do sexto nervo através de vídeos. Para isso, utilizou-se a rede neural convolucional YOLO para detecção da região dos olhos, e a partir da região detectada, utilizou-se a técnica da curvatura de Isophotes para identificar o centro da pupila. Esse processo foi aplicado em todos os quadros dos vídeos utilizados, a fim de manter um registro da movimentação dos olhos em um intervalo de tempo. A partir desse registro, calculou-se a velocidade média do deslocamento de cada olho. Ao analisar os valores da velocidade média referentes à olhos que possuem a paralisia, verificou-se que eles se movem de forma bem mais lenta que um olho saudável, apresentando quase a metade da velocidade de um olho que não possui a paralisia (em média $42 \%$ mais lentos). Apesar dos resultados terem mostrado 


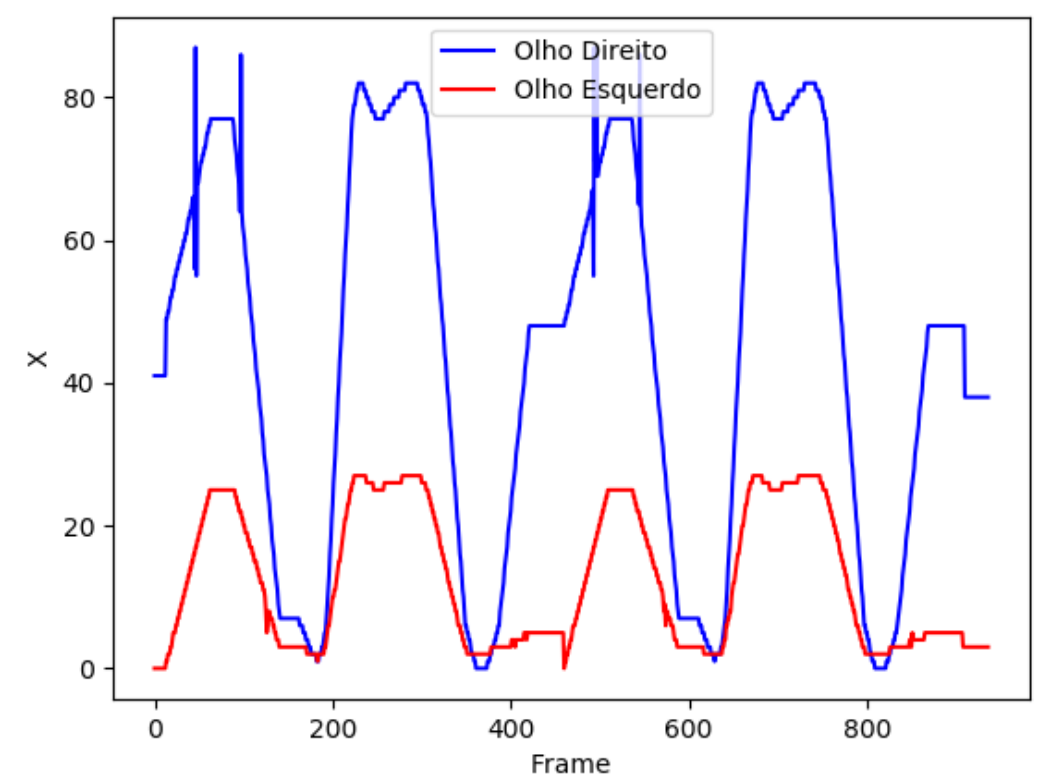

Figura 5. Trajetória do deslocamento no eixo X de cada olho. (Referente ao vídeo 8)

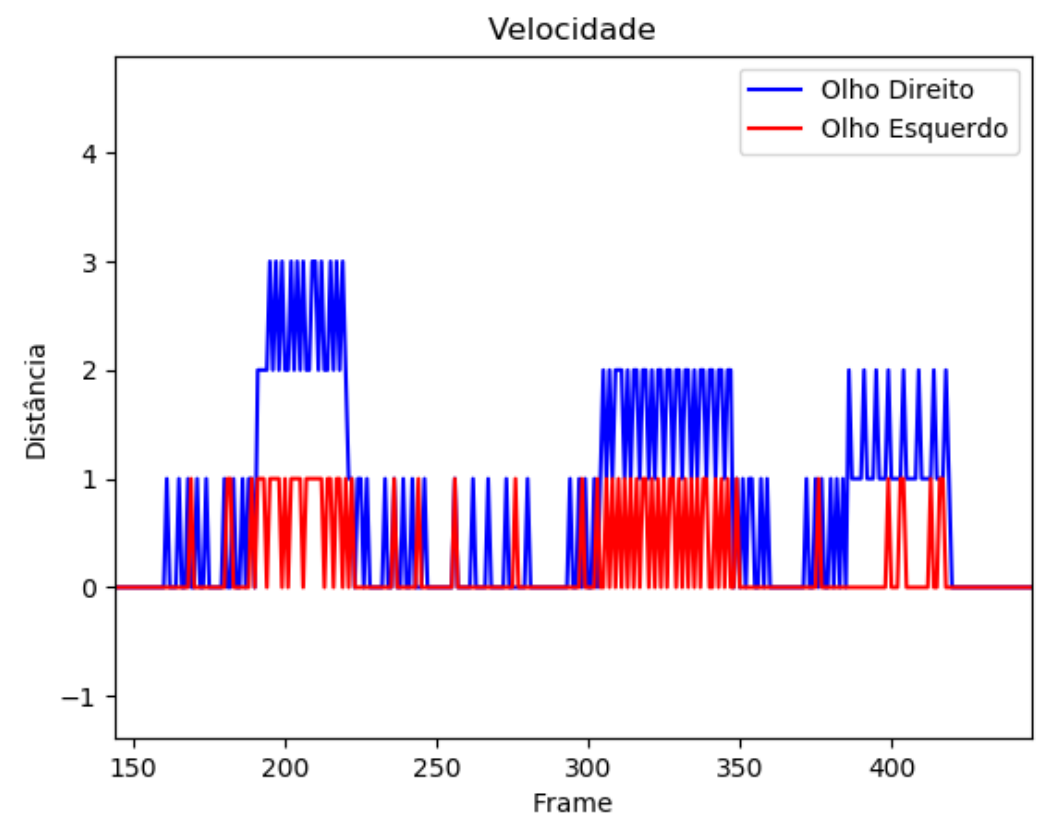

Figura 6. Velocidade de cada olho dada em pixels por quadro. (Referente ao vídeo 8)

que o método é promissor, a pequena base de vídeos dificulta a validação proposta. Portanto, como trabalho futuro, pretende-se aumentar a base dados, incluindo mais vídeos de pacientes saudáveis, além de definir um protocolo de aquisição dos vídeos, a fim de evitar ruídos na aquisição dos dados. Além disso, apesar da rede YOLO funcionar em tempo 
real, a combinação com a técnica de Isophotes tornou o método lento, portanto, visa-se incluir técnicas de rastreamento de objetos a fim de que o método proposto funcione em tempo real.

Por envolver o estudo com seres humanos, este trabalho foi submetido para avaliação por um Comitê de Ética em Pesquisa e aguarda aprovação, a fim de seguir as diretrizes do Conselho Nacional de Saúde dispostas na Resolução n ${ }^{\circ}$ 196/96.

O presente trabalho foi realizado com apoio da Coordenação de Aperfeiçoamento de Pessoal de Nível Superior - Brasil (CAPES) - Código de Financiamento 001 e do Conselho Nacional de Desenvolvimento Científico e Tecnológico (CNPQ) - Processo 307210/2018-9.

\section{Referências}

Curi, R. L. N., de Oliveira Costa, I. C. B., and Barroso, T. G. M. (2013). Paralisia do vi nervo (abducente). Rev Bras Oftalmol, 72(1):59-69.

Deng, J., Dong, W., Socher, R., Li, L.-J., Li, K., and Fei-Fei, L. (2009). Imagenet: A large-scale hierarchical image database.

Ehrenhau, M. P. (2018). Abducens nerve palsy (sixth cranial nerve palsy).

GARD (2015). Sixth nerve palsy.

Gonzalez, R. C. and Woods, R. E. (2007). Image processing. Digital image processing, 2.

Gray, H. and Goss, C. M. (1974). Anatomy of the human body. American Journal of Physical Medicine \& Rehabilitation, 53(6):293.

Martinsen, O. G. and Grimnes, S. (2011). Bioimpedance and bioelectricity basics. Academic press.

Ouabida, E., Essadique, A., and Bouzid, A. (2017). Vander lugt correlator based active contours for iris segmentation and tracking. Expert Systems with Applications, 71:383395.

Powers, D. M. (2011). Evaluation: from precision, recall and f-measure to roc, informedness, markedness and correlation.

Redmon, J., Divvala, S., Girshick, R., and Farhadi, A. (2016). You only look once: Unified, real-time object detection. In Proceedings of the IEEE conference on computer vision and pattern recognition, pages 779-788.

Redmon, J. and Farhadi, A. (2018). Yolov3: An incremental improvement. arXiv preprint arXiv:1804.02767.

Resnick, R., Halliday, D., and WALKER, J. (2001). Fundamentos de física. Grupo Patria Cultural SA DE CV.

Rosebrock, A. (2016). Intersection over union (iou) for object detection.

Rubin, M. and Cornell, W. (2018). Paralisia do sexto nervo craniano (nervo motor ocular externo).

Szegedy, C., Ioffe, S., Vanhoucke, V., and Alemi, A. A. (2017). Inception-v4, inceptionresnet and the impact of residual connections on learning. In $A A A I$, volume 4, page 12. 
Trade, P. (2018). A keras implementation of yolov3.

Valenti, R. and Gevers, T. (2008). Accurate eye center location and tracking using isophote curvature. 